\title{
Recent Results on Fixed Point Approximations and Applications
}

\author{
Jong Kyu Kim, ${ }^{1}$ Poom Kumam, ${ }^{2}$ Xiaolong Qin, ${ }^{3}$ and Kyung Soo Kim ${ }^{4}$ \\ ${ }^{1}$ Department of Mathematics Education, Kyungnam University, Changwon, Gyeongnam 631-701, Republic of Korea \\ ${ }^{2}$ Department of Mathematics, Faculty of Science, King Mongkut's University of Technology Thonburi (KMUTT), \\ Bang Mod, Thrung Kru, Bangkok 10140, Thailand \\ ${ }^{3}$ Department of Mathematics and Applied Mathematics, Hangzhou Normal University, Hangzhou 310036, China \\ ${ }^{4}$ Graduate School of Education, Mathematics Education, Kyungnam University, Changwon, \\ Gyeongnam 631-701, Republic of Korea \\ Correspondence should be addressed to Jong Kyu Kim; jongkyuk@kyungnam.ac.kr
}

Received 28 May 2015; Accepted 30 May 2015

Copyright (C) 2015 Jong Kyu Kim et al. This is an open access article distributed under the Creative Commons Attribution License, which permits unrestricted use, distribution, and reproduction in any medium, provided the original work is properly cited.

The aim of this special issue is to promote research and its applications in the area of nonlinear functional analysis and applications. It will reflect theoretical research and advanced applications. One of the most important and significant areas is fixed point theory being very rich, interesting, and extremely applicable area of mathematics and mathematical sciences.

In the last three decades, the problems of nonlinear analysis with its relation to fixed point theory have emerged as a rapidly growing area of research because of its applications in differential equation, KKM theory, nonlinear ergodic theory, game theory, optimization problem, control theory, and so on. Also, the iterative methods for finding the approximate solutions of fixed point problems, variational inequality problems, equilibrium problems, optimization problems, split feasibility problems, operator equations and inclusion problems, amenability of semigroup, and convergence of iterative approximations are very important and useful.

\section{Acknowledgments}

As guest editors for this special issue, we wish to thank all those who submitted manuscripts for publication and many mathematicians who served as the reviewers. We hope that all the papers which are published in this special issue can be motivated for the development of research works of the researchers.

Jong Kyu Kim

Poom Kumam

Xiaolong Qin

Kyung Soo Kim 


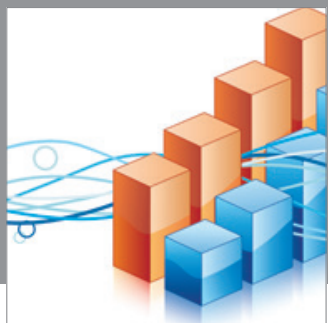

Advances in

Operations Research

mansans

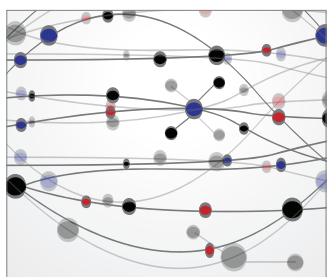

The Scientific World Journal
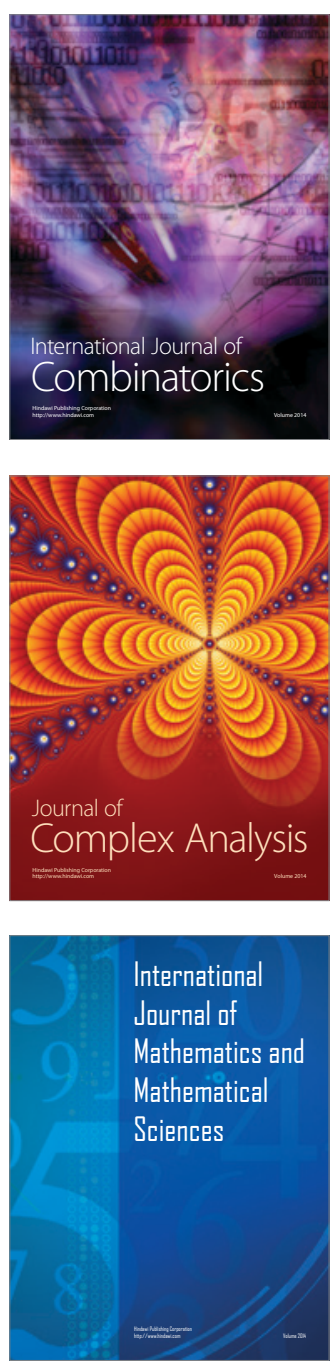
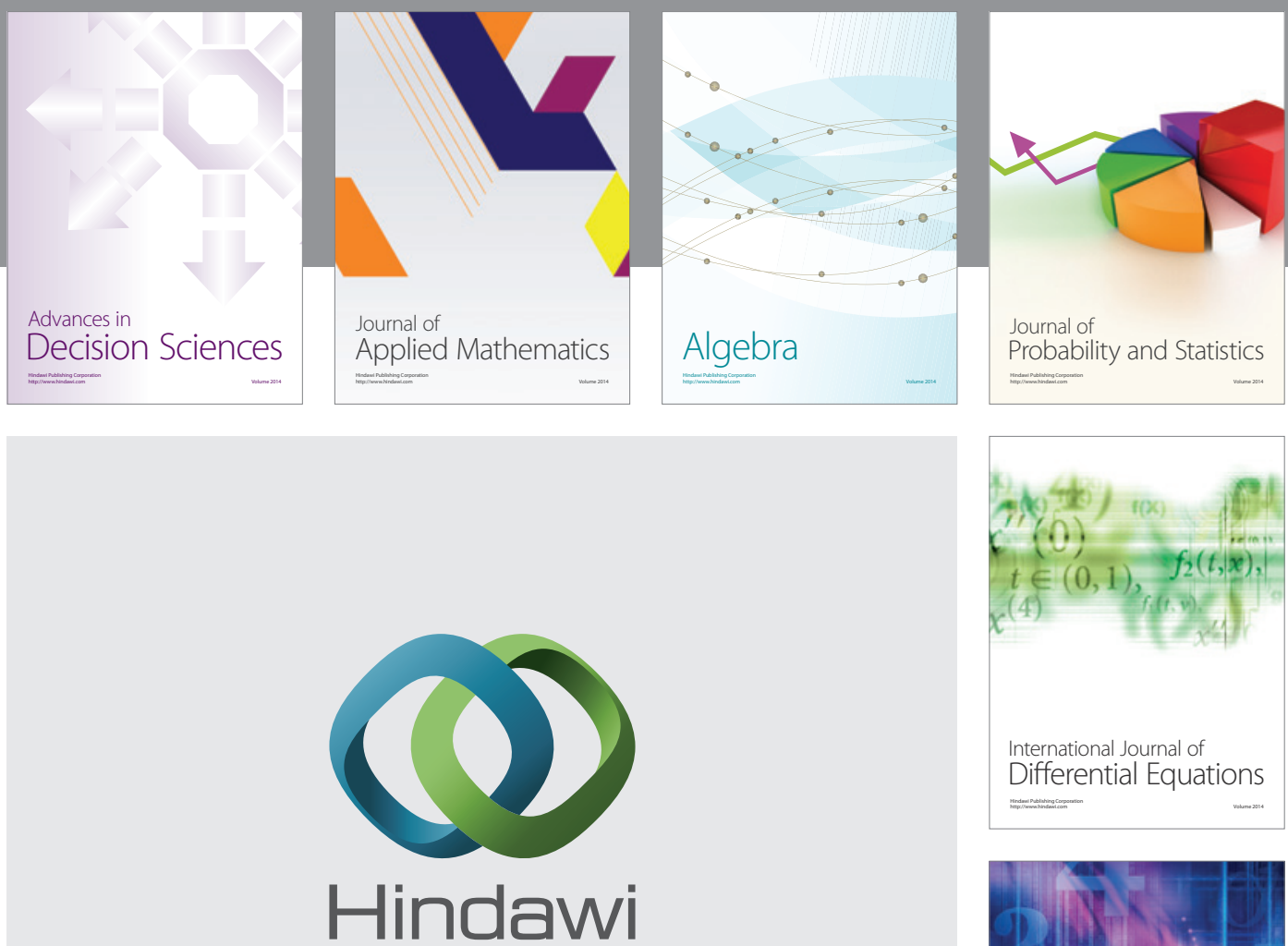

Submit your manuscripts at http://www.hindawi.com
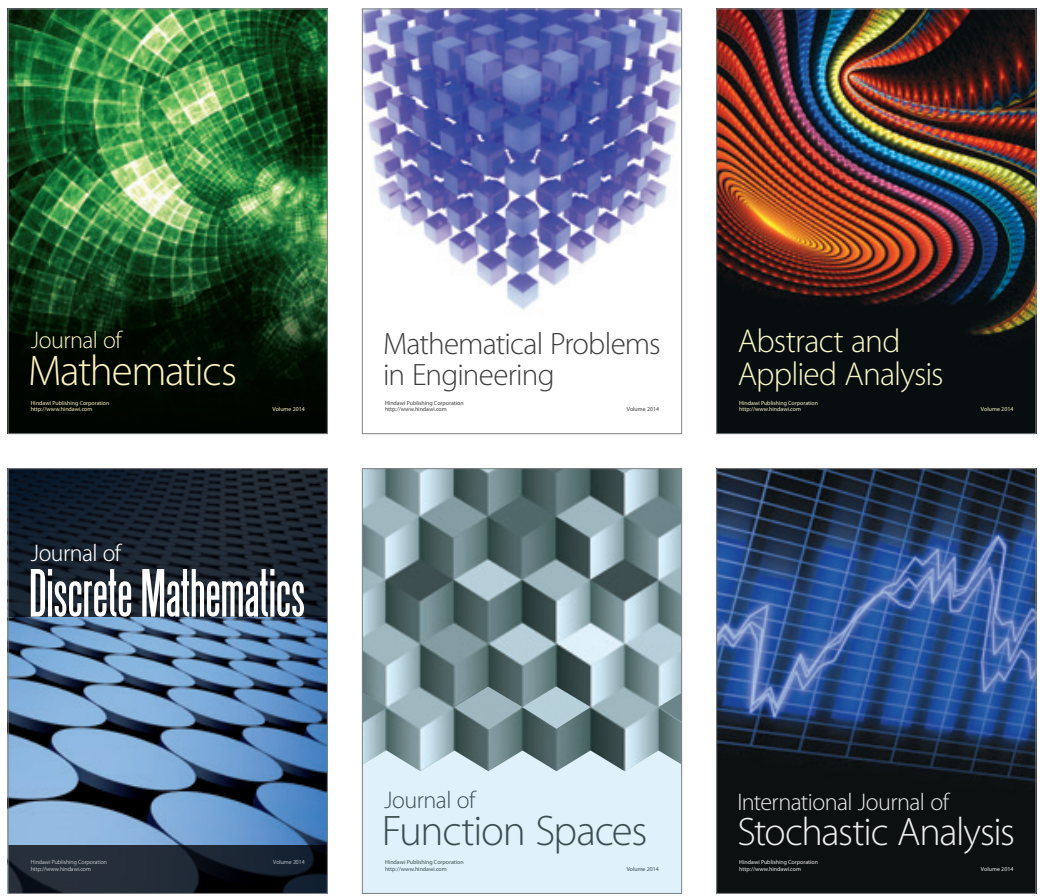

Journal of

Function Spaces

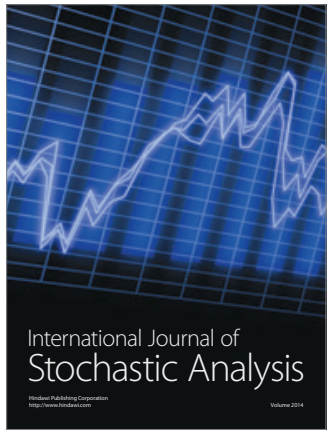

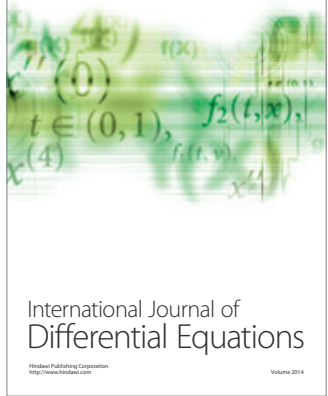
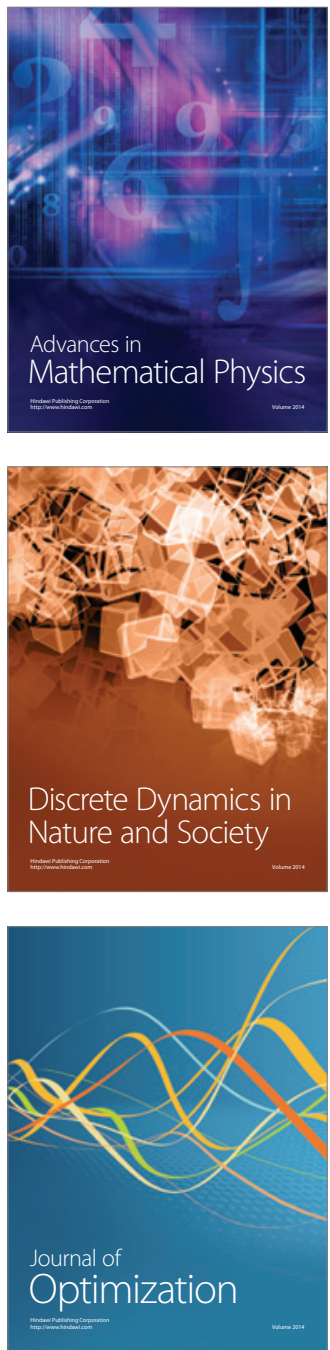